\title{
Space-filling curves for 2-simplicial meshes created with bisections and reflections
}

\author{
Dr. Joseph M. Maubach* \\ Department of Mathematics \\ Eindhoven University of Technology \\ Eindhoven, The Netherlands \\ j.m.l.maubach@tue.nl
}

January 17,2005

\section{Abstract}

Numerical experiments in J. Maubach, Local bisection refinement and optimal order algebraic multilevel preconditioners, PRISM-97 conference Proceedings (eds. O. Axelsson et al), University of Nijmegen, the Netherlands, 1997, 121-136 indicated that the refinement with the use of local bisections presented in J. Maubach, Local bisection refinement for n-simplicial grids generated by reflections, SIAM Journal on Scientific Computing, 16(1995), 210-227 leads to highly locally refined computational 2-meshes which can be very efficiently load-balanced with the use of a space-filling curve. This paper introduces the construction of this curve, which can be produced at almost no costs, proofs that all its properties are invariant under the local bisection, and comments at the 3 -dimensional case.

Keywords: Grid generation, space filling curve, load balancing

Mathematics Subject Classification 2000: 65M50

${ }^{*}$ Part of this work was supported by a N.W.O. grant 047.016 .008 


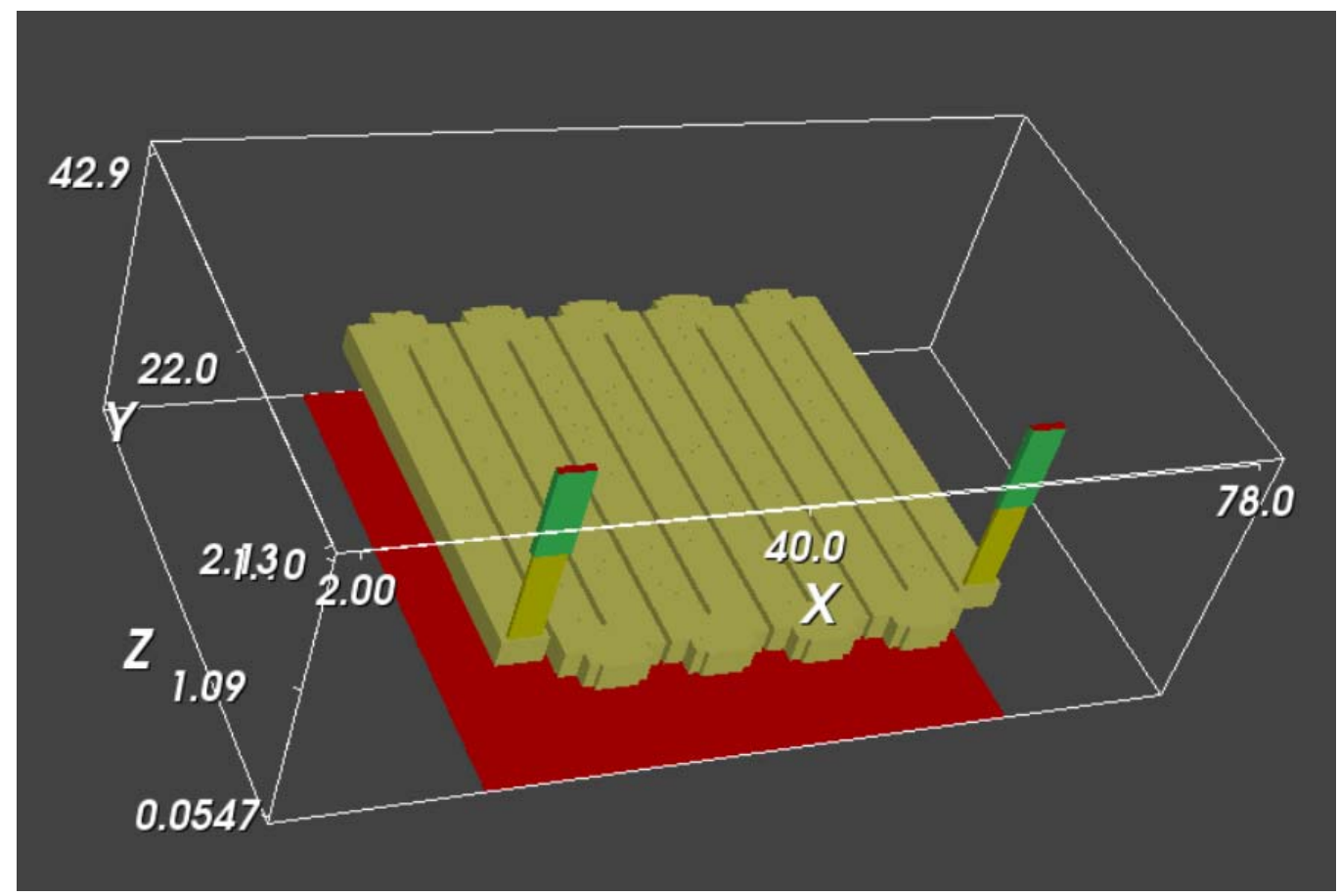

Figure 1: An on-chip resistor.

\section{Introduction}

Refinement with the use of local bisection is examined and discussed in [1], [13], [6], [8] and [7], [3], [2], [12] and [11]. The local bisection refinement in [6] is simplest and efficient, and it is suited for modern challinging applications such as optics and on-chip-interconnects (figure 1 shows an on-chip resistor). In all such applications, the domains of interest are a coarse tensor product mesh refined with the use of local bisection. These challinging applications require massive parallel calculations (even massive parallel methods such as in [4], [5]), which at their turn require a load balance algorithm.

This paper complements the local bisection method in [6] with a local curve adaptation method such that, independent on the level of refinement, the curve traverses traverses all 2-simplices ones and does not intersect/touch itself (see (P1) and (P2) in section 3). The fact that one such curve traverses all elements through midpoints of facets without selfintersection is not 


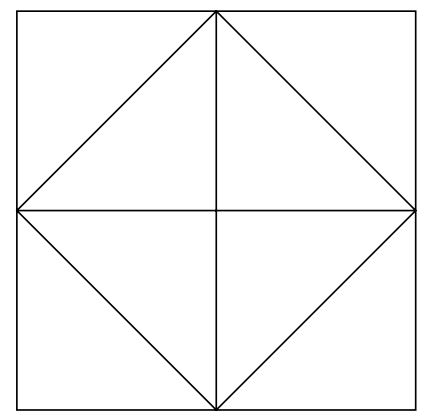

Figure 2: A single non-intersecting curve is not always possible.

trivial, because some simple meshes will not permit one curve (see figure 2). Furthermore, the manner in which the curve traverses all simplices (possible descendents created by refinement is special (see (P1)): The amount of vertices at the border of a subdomain will be small: Fast communication.

The local curve adaptation method uses but the stored $n$-simplex data (coordinates of the 3 vertices or levels of the 1-facets) and hence comes at neglegible costs. The curve adaptation method is defined with the introduced concept of "the level of a 1-facet". The different experimentally guessed adaptation method presented in [9] produces identical curves.

The curve can be used to put the elements in curve-traversal order, and the required load-balance redistribution amounts to a possible inexpensive move of some head-part- or tail-part-elements to another processor.

Section 3 presents the bisection method [6], introduces the concept of clusters and facet-levels, defines the curve per element and proofs that it remains connected and non-intersects under refinement. Then, section 4 discusses expectations for the 3-dimensional case, and section 5 formulates the conclusions.

\section{The local curve adaptation method}

This section discusses the construction of the curve. First, we introduce the bisection method from [6], and proof that compatible 2-elements always turn 

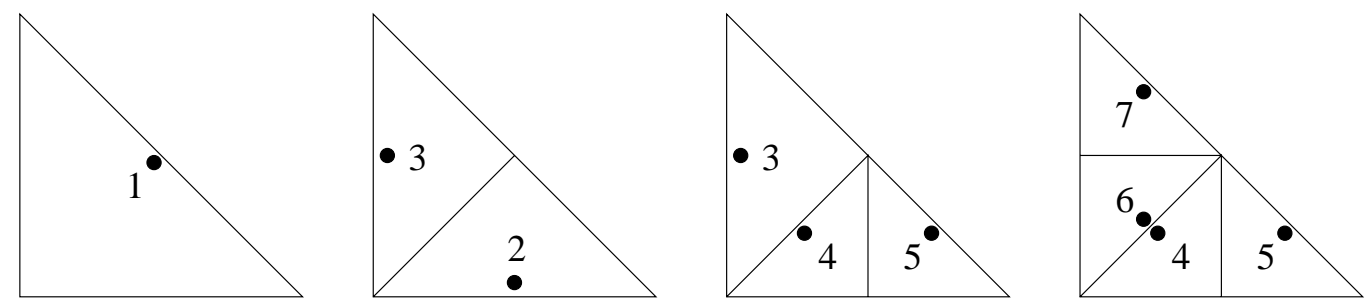

Figure 3: Three subsequent bisection steps.

out to be also elements of one of a sequence of uniform meshes. The related lines of symmetry are assigned a unique level, and 1-facets inherit the level of the line on which they are situated. Then, the curve per element is defined with the use of these levels. Note that not all meshes permit a spacefilling curve (see figure 2), this paper just proofs that meshes created with the local bisection method presented in [6] do so.

The local refinement presented in [6], starts from a coarse mesh $\Omega_{1}$ (see figure 5) where elements are all similar under reflection (horizontal and vertical lines) and permutations (skew lines). Then applied local bisections lead to a refinened mesh (see figure 4 ).

Each local bisection involves one or two elements, which share the 1-facet to be bisected $([6])$. Each of these elements is bisected into 2 descendents (also called its children). As an example, consider figure 3. Here one element is bisected multiple times, which leads to four descendents. For each numbered elements, the 1-facet to be bisected is marked with a black bullet. By construction (see [6]) elements 4 and 6 are compatible, but 3 and 4 are not. Figure 5) shows collections of compatible elements $(A-E)$.

To start with, we need the following property, which was not proven in [6] because this paper restricts itself to the general $n$-dimensional case.

Lemma: All 2-elements in a locally refined mesh have their to be bisected 1-facet opposite the $\pi / 4$ angle.

Proof: Figure 3 shows that this holds under bisection (these are all cases in two dimensions, see [6]). The property holds for all four elements of $\Omega_{1}$ in 

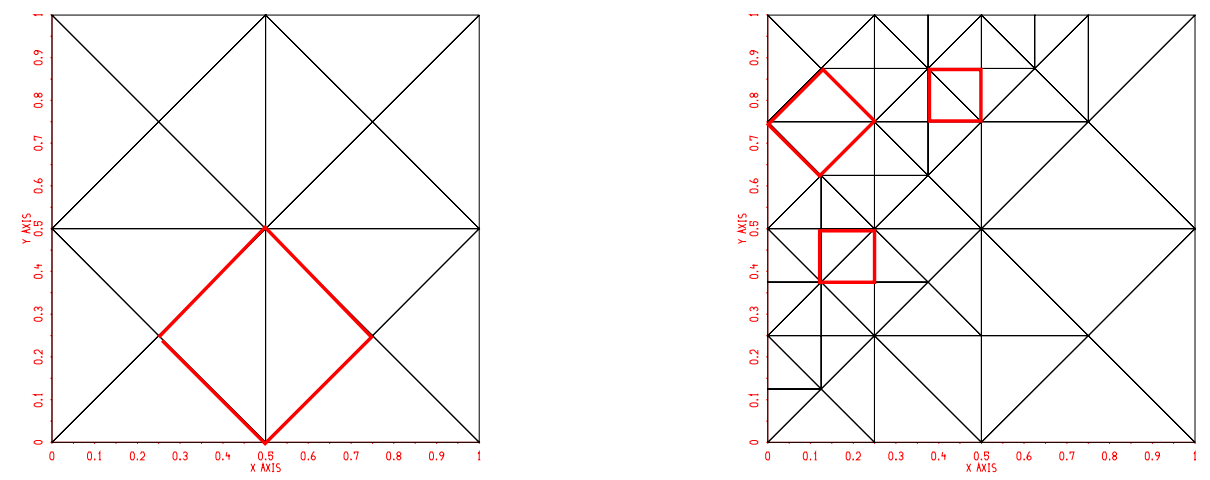

Figure 4: The coarse mesh and the refined mesh.

figure 5 (see [6]). Hence, by induction under refinement, the property holds for all elements in a locally refined mesh. $\diamond$

For the sake of brevity, we use the word cluster for compatible pair of to be bisected 2-elements. Note that a cluster in a refined mesh (see figure 4) is also a cluster $\mathrm{A}-\mathrm{D}$ in a uniform mesh (see figure 5). The vertices of elements of a uniform mesh are on a lattice (are on regularly spaced positions) whence also the vertices of an element in a cluster are on a lattice. The $x$ and $y$-coordinates of each lattice are of the from $k / 2^{L}$ for $L>0$ and $k \in\left\{0,1, \ldots, 2^{L}-1\right\}$ and its lines of symmetry are either $y=x, y=1-x$, $y=0$ and $x=0$ or images under a shift of the form $k / 2^{L}$ for $L \geq 0$ and $k \in\left\{0,1, \ldots, 2^{L}-1\right\}$.

Our space-filling curve construction for a cluster of elements in a refined mesh will make use of the horizontal and skew lines in the lattice which contains this cluster, as follows.

Definition: Let $k \bmod 2^{L}=1$ or $L=0$. The level of each line in the lattice $\Omega_{1}, \Omega_{2}, \ldots, \Omega_{L}, \ldots$ in figure 5 are: 

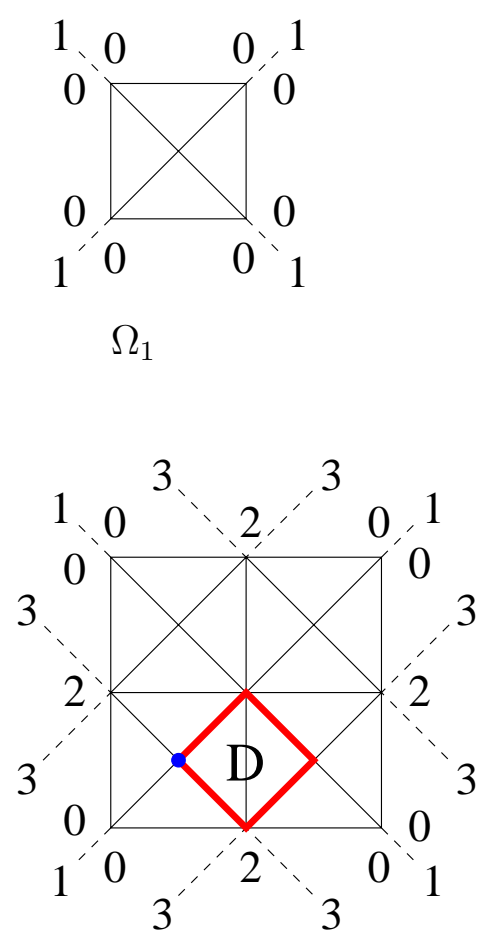

$\Omega_{3}$
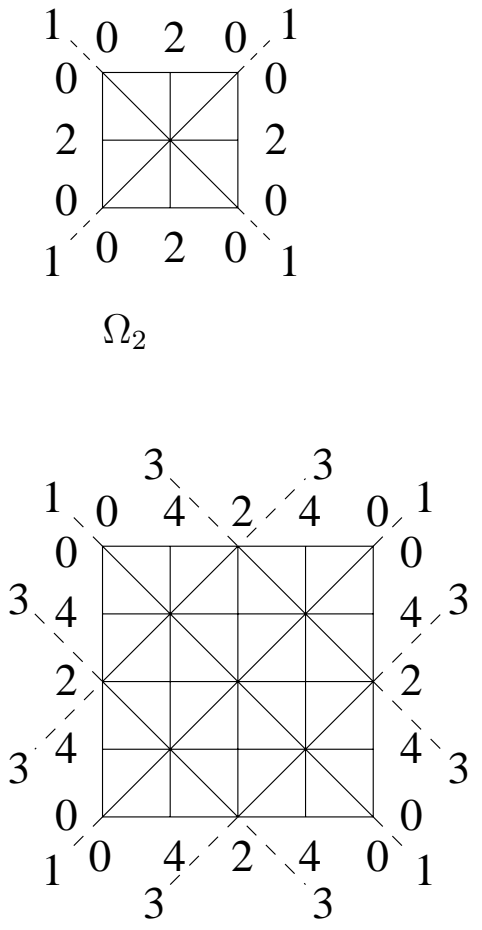

$\Omega_{4}$
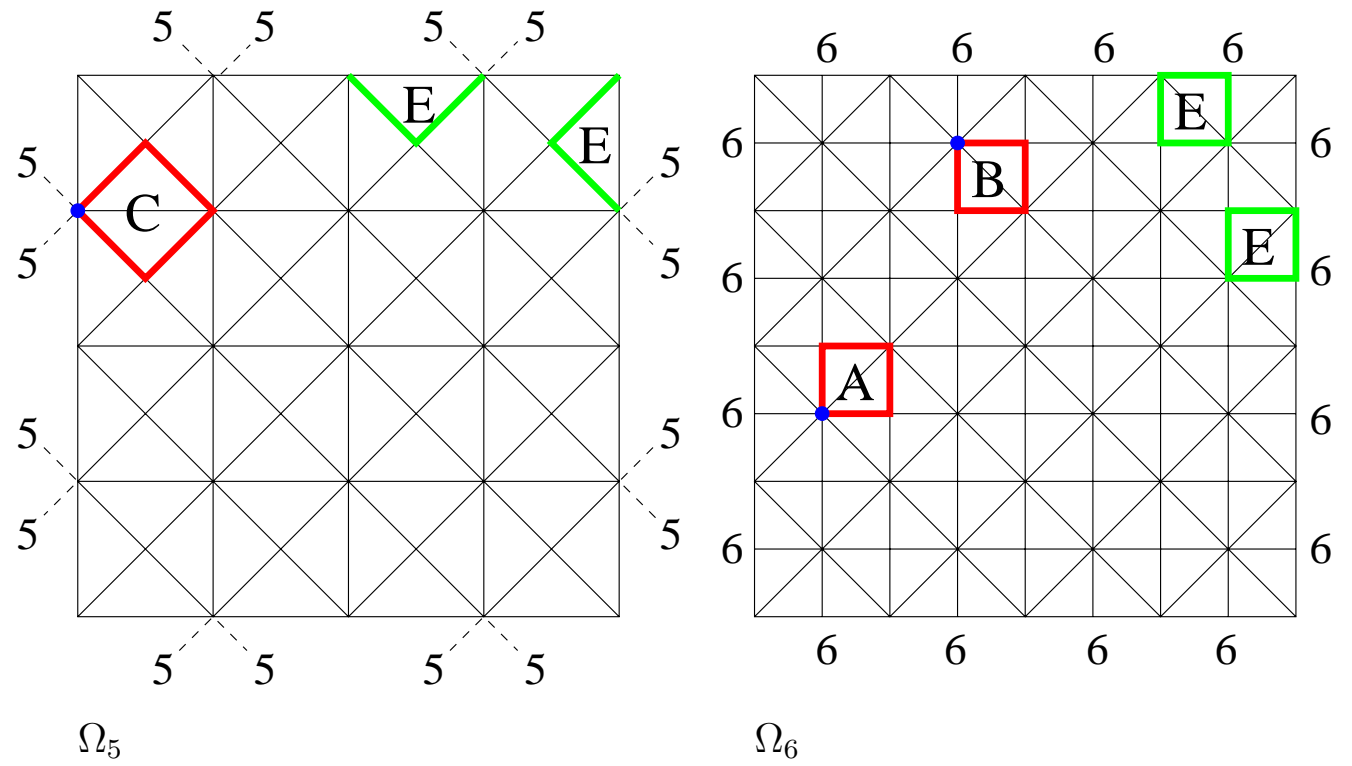

Figure 5: The sequence of uniform refined meshes (lattices). 


\begin{tabular}{|c|c|}
\hline Line & level \\
\hline$y=x+k / 2^{L}$ & level $2 L+1$ \\
$y=1-x+k / 2^{L}$ & level $2 L+1$ \\
$y=k / 2^{L}$ & level $2 L$ \\
$x=k / 2^{L}$ & level $2 L$ \\
\hline
\end{tabular}

Lattice line levels.

Note that the highest level line in lattice $\Omega_{L}$ is $L$ and that the bisection of a cluster in $\Omega_{L}$ creates a new 1 -facet on a line of level $L+1$. An element in $\Omega_{L}$, has vertices with coordinates of the form

$$
\frac{k}{2^{\lceil L / 2\rceil},}
$$

where $\lceil$.$\rceil stands for round upwards to the nearest integer value. Further-$ more observe that each element of $\Omega_{L}$ has at least one horizontal/vertical 1-facet of level $L$.

Definition: Let the 2-element in a (refined) mesh be an element in one of the uniform meshes $\Omega_{L}$. Then the level of one of its 1-facets is equal to the level of the line which contains this facet.

Definition: For each 2-element in the (refined) mesh, its curve connects the midpoints of its two highest-level 1-facets.

Based on the level of the edges of $\Omega_{1}$ (see figure 5 ), the related curve is a multi-line through $(1 / 4,1 / 4),(3 / 4,1 / 4),(3 / 4,3 / 4),(1 / 4,3 / 4)$, which is connected and non-self-intersecting. Left to be proven is that

(P1) subsequently traverses both children;

(P2) remains connected (does not intersect/touch itself);

remain invariant under bisection. In order to show this, we distinguish 5 cases $\mathrm{A}-\mathrm{E}$, see figure 5 . The cases $\mathrm{A}$ and $\mathrm{B}$ are all different cluster orientations in $\Omega_{L}$ with $L$ even, the cases $\mathrm{C}$ and $\mathrm{D}$ all cluster orientation in $\Omega_{L}$ with $L$ odd, and the cases $E$ are all cases of clusters from A - D with one or more 1-facets at one of the boundaries:

Lemma: There are (apart from translation) 5 different cluster orientations 

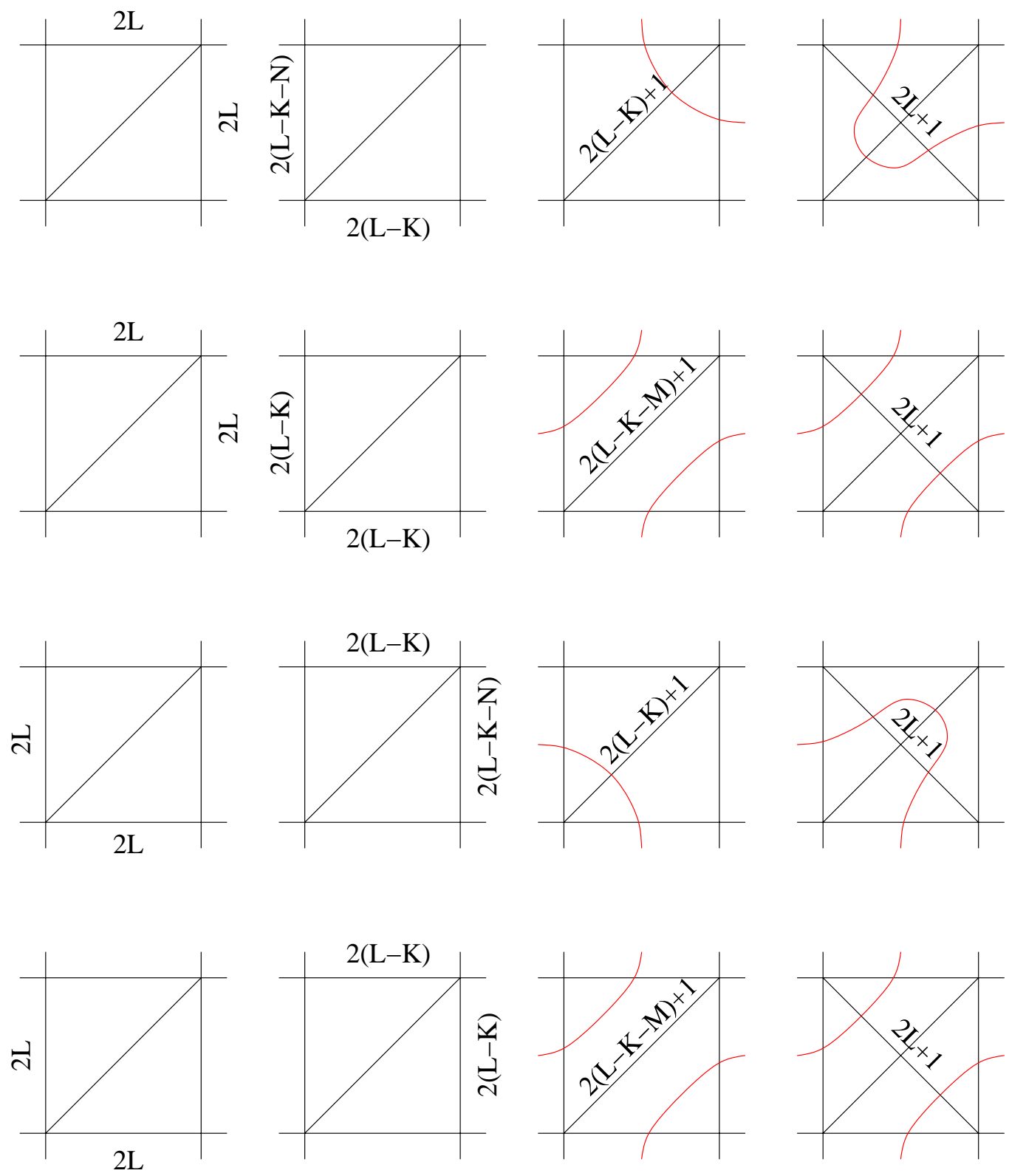

Figure 6: Orientations A: Local bisection keeps the curve connected. 

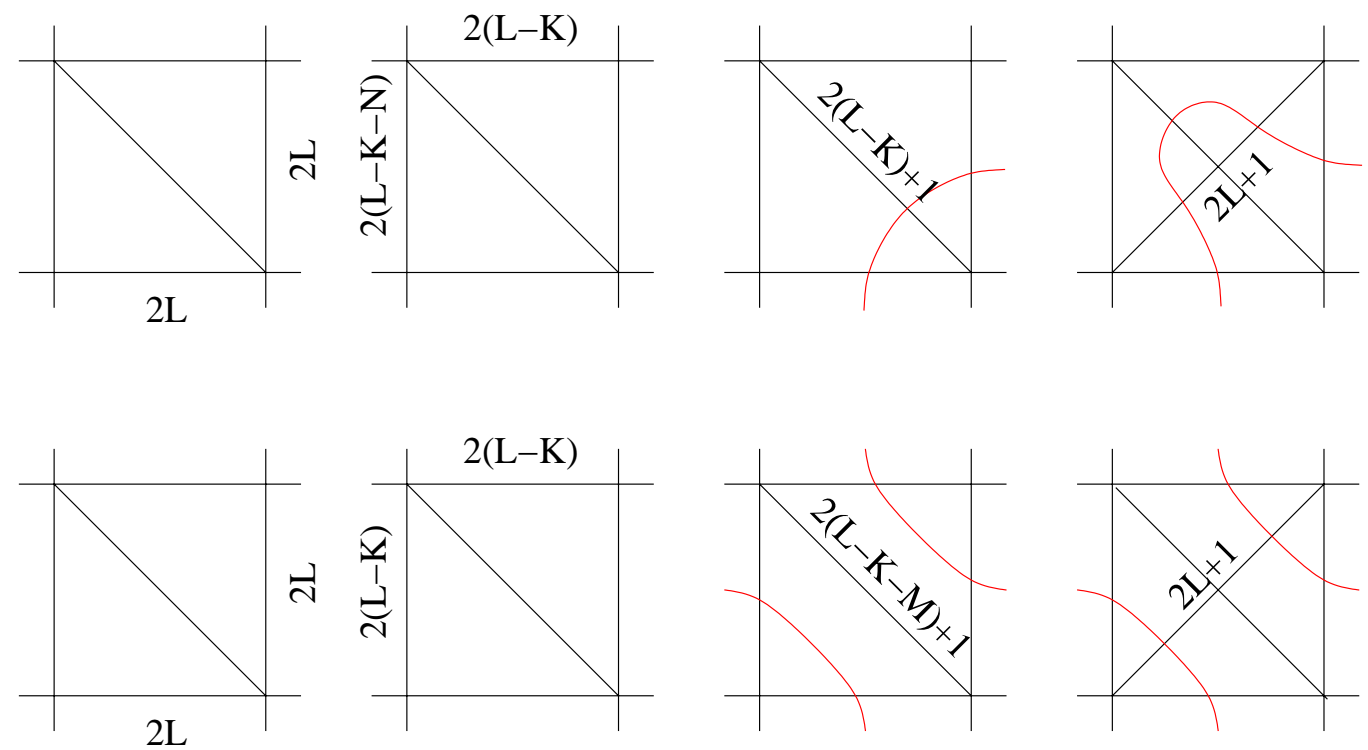

Figure 7: Orientations B: Local bisection keeps the curve connected.

on each lattice $\Omega_{1}, \Omega_{2}, \ldots$, called A, B, C and D. Clusters at the boundaries are called E. $\diamond$

Lemma: The bisection of a cluster of orientation A satisfies (P1) and (P2).

Proof: From the coordinates of one of the vertices of the cluster, by and by, we determine all levels of its 1-facets (first three columns of figure 6). Based on these levels, we show how the curve traverses the cluster respectively bisected cluster (last two columns of figure 6).

Note that a cluster of orientation $\mathrm{A}$ on is on a lattice of the form $\Omega_{2 \cdot L}$. It has a base point (shown in figure 5) of the form $\left(x_{b}, y_{b}\right)=\left(k / 2^{L}, l / 2^{L}\right)$, where $\bmod (k-l, 2)=0$. We will distinghuish 2 cases, $k$ is even and $k$ is odd.

First assume $k$ is even, the case depicted in the first row of figure 6. Now, because $k$ is even and $\bmod (k-l, 2)=0$, also $l$ is even. From this follow the first two levels of 1 -facet, shown in the first column of figure 6 (recall, one of the horizontal and one of the vertical lines are of level $2 L$ ). 
Now assume $x \geq y$ (other case $y \geq x$ can be treated indentically). Then we know that there exist a $K>0, N \geq 0$ and odd numbers $x_{\text {odd }}$ and $y_{\text {odd }}$ such that we can factor:

$$
x_{b}=\frac{2^{K} \cdot 2^{N} \cdot x_{\text {odd }}}{2^{L}}, \quad y_{b}=\frac{2^{K} \cdot y_{\text {odd }}}{2^{L}} .
$$

First assume that $N>0$ ( $k$ still even). Then from

$$
x_{b}=\frac{x_{\text {odd }}}{2^{L-K-N}}, \quad y_{b}=\frac{y_{\text {odd }}}{2^{L-K}}
$$

we obtain the level of the other two horizonal/vertical 1-facets (2-nd column of the first row in 6). For the diagonals level, observe that the diagonal is a line of the form

$$
y=x+y_{b}-x_{b},
$$

where the difference $y_{b}-x_{b}$ determines the level:

$$
y_{b}-x_{b}=\frac{2^{K} 2^{N} x_{\text {odd }}}{2^{L}}-\frac{2^{K} y_{\text {odd }}}{2^{L}}=\frac{2^{N} x_{\text {odd }}}{2^{L-K}}-\frac{y_{\text {odd }}}{2^{L-K}}=\frac{z_{\text {odd }}}{2^{L-K}},
$$

with $z_{\text {odd }}$ is odd (difference between an even and an odd number) which (see table with lattice line levels) implies that our diagonal's level is $2(L-K)+1$, depicted in the 3 -rd column of the first row in 6 . Because the 1-facet created in the bisection step will be of level $2 L+1$ we can depict the form of the curve in the last 2 columns of the first row of 6 . This shows that the curve subsequently traverses both children, and that it remains connected under the bisection step. This concludes that case (2) for $N>0$.

Now assume that $N=0$ ( $k$ still even). This determines the levels of the other two horizonal/vertical 1-facets (2-nd column of the second row in figure 6 (where now $N=0$ ). The level of the diagonal can be determined from:

$$
y_{b}-x_{b}=\frac{2^{K} x_{\text {odd }}}{2^{L}}-\frac{2^{K} y_{\text {odd }}}{2^{L}}=\frac{x_{\text {odd }}}{2^{L-K}}-\frac{y_{\text {odd }}}{2^{L-K}}=\frac{2^{M} z_{\text {odd }}}{2^{L-K}}=\frac{z_{\text {odd }}}{2^{L-K-M}}
$$

where $z_{\text {odd }}$ is an odd number, and $M>0$. Hence, here the level of the diagonal is $2(L-K-M)+1$, (3-rd column of the second row in figure 6$)$ and (P1) and (P2) hold for the related curves in the last to columns of figure 6 . 
To finish with, consider the case where $k$ is odd. Also here assume that $x_{b} \geq y_{b}$. This proof follows in the same manner. The first two levels (1-st column of the 3-rd and 4-th row in figure 6) are known because

$$
x_{b}=\frac{x_{\text {odd }}}{2^{L}}, \quad y_{b}=\frac{y_{\text {odd }}}{2^{L}} .
$$

The other two horizontal/vertical lines' and the diagonal's level follow best from the top right vertex of the cluster,

$$
\left(x_{b}^{\prime}, y_{b}^{\prime}\right)=\left(\frac{1+x_{\mathrm{odd}}}{2^{L}}, \frac{1+y_{\mathrm{odd}}}{2^{L}}\right) .
$$

where $1+x_{\text {odd }}$ and $1+y_{\text {odd }}$ are even. Now we follow the line of the $k$ even case: It follows that there exist a $K>0, N \geq 0$ and odd numbers $x_{\text {odd }}$ and $y_{\text {odd }}$ such that we can factor:

$$
x_{b}^{\prime}=\frac{2^{K} \cdot 2^{N} \cdot x_{\mathrm{odd}}}{2^{L}}, \quad y_{b}^{\prime}=\frac{2^{K} \cdot y_{\mathrm{odd}}}{2^{L}} .
$$

which determines the two levels shown in the 2-nd column of the last two rows in figure 6. For the level of the diagonal, observe that for $N>0$ :

$$
y_{b}^{\prime}-x_{b}^{\prime}=\frac{2^{K} 2^{N} x_{\text {odd }}}{2^{L}}-\frac{2^{K} y_{\text {odd }}}{2^{L}}=\frac{2^{N} x_{\text {odd }}}{2^{L-K}}-\frac{y_{\text {odd }}}{2^{L-K}}=\frac{z_{\text {odd }}}{2^{L-K}},
$$

and that for $N=0$ :

$$
y_{b}^{\prime}-x_{b}^{\prime}=\frac{2^{K} x_{\text {odd }}}{2^{L}}-\frac{2^{K} y_{\text {odd }}}{2^{L}}=\frac{x_{\text {odd }}}{2^{L-K}}-\frac{y_{\text {odd }}}{2^{L-K}}=\frac{2^{M} z_{\text {odd }}}{2^{L-K}}=\frac{z_{\text {odd }}}{2^{L-K-M}},
$$

which leads to the 3 -rd column of the 3 -rd respectively 4 -th row in figure 6 . $\diamond$.

Note: The proof that the curve satisfies all properties for cluster orientation A relies on the fact that all levels of all 1-facets can be calculated from one of the cluster's vertices $\left(x_{b}, y_{b}\right)=\left(k / 2^{L}, l / 2^{L}\right)$. The amount of factors 2 in $k$ determines the level of the vertical 1 -facets, that amount in $l$ determines the level of the horizontal 1-facets, and that in $k-l$ the level of the diagonal.

Lemma: The bisection of a cluster of orientation $\mathrm{B}-\mathrm{E}$ satisfies $(\mathrm{P} 1 / 2)$. 

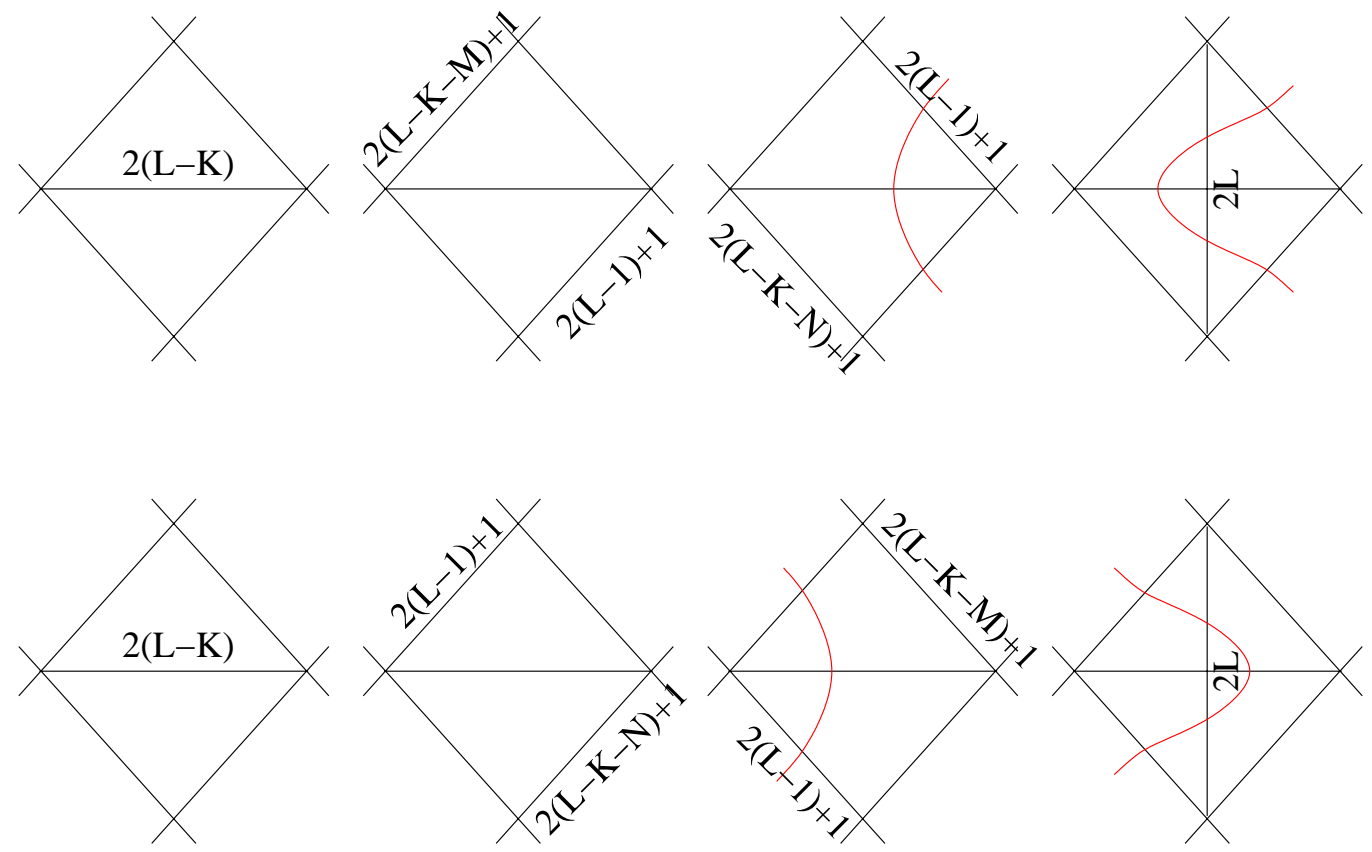

Figure 8: Orientations C: Local bisection keeps the curve connected.

Proof: Also here, all levels can be calculated from the amount of factors 2 in $k$ and $l$. For case B, use vertex $\left(x_{b}, y_{b}\right)=\left(k / 2^{L}, l / 2^{L}\right)$ shown in figure 5 and proceed as in case A (also distinguish $k$ is odd and even). Also here either $k$ and $l$ have the same amount of factors 2 (row 2 figure 7 ) whence the $l-k$ has extra factors $2(M>0)$, or $k$ and $l$ have a different amount of factors of 2 (row 2 figure 7$)(N>0)$ whence $l-k$ has no extra factors of 2 .

The case C: Here $\left(x_{b}, y_{b}\right)=\left(k / 2^{L}, l / 2^{L}\right)$ is such that $k$ and $l$ are both even or both odd. Two of the diagonals must be of level $2(L-1)+1$. Also here the count of factors 2 leads to the desired results: If $k$ and $l$ have an identical amount of factors 2 then $k-l$ and $k+l+2^{L}$ have extra factors 2, which explains the $M>0$ and $N>0$ in columns 2 and 3 of row 1 of figure 8 . The other case where $k$ and $l$ have different amounts of factor 2 needs more attention: Either $K=1$ (which is the minimum) and we end up in the 2-nd row of figure 8 , or $K>1$ and we end up in the 1-st row of this figure. The case $\mathrm{D}$ can be treated in an identical manner to the case $\mathrm{C}$. 

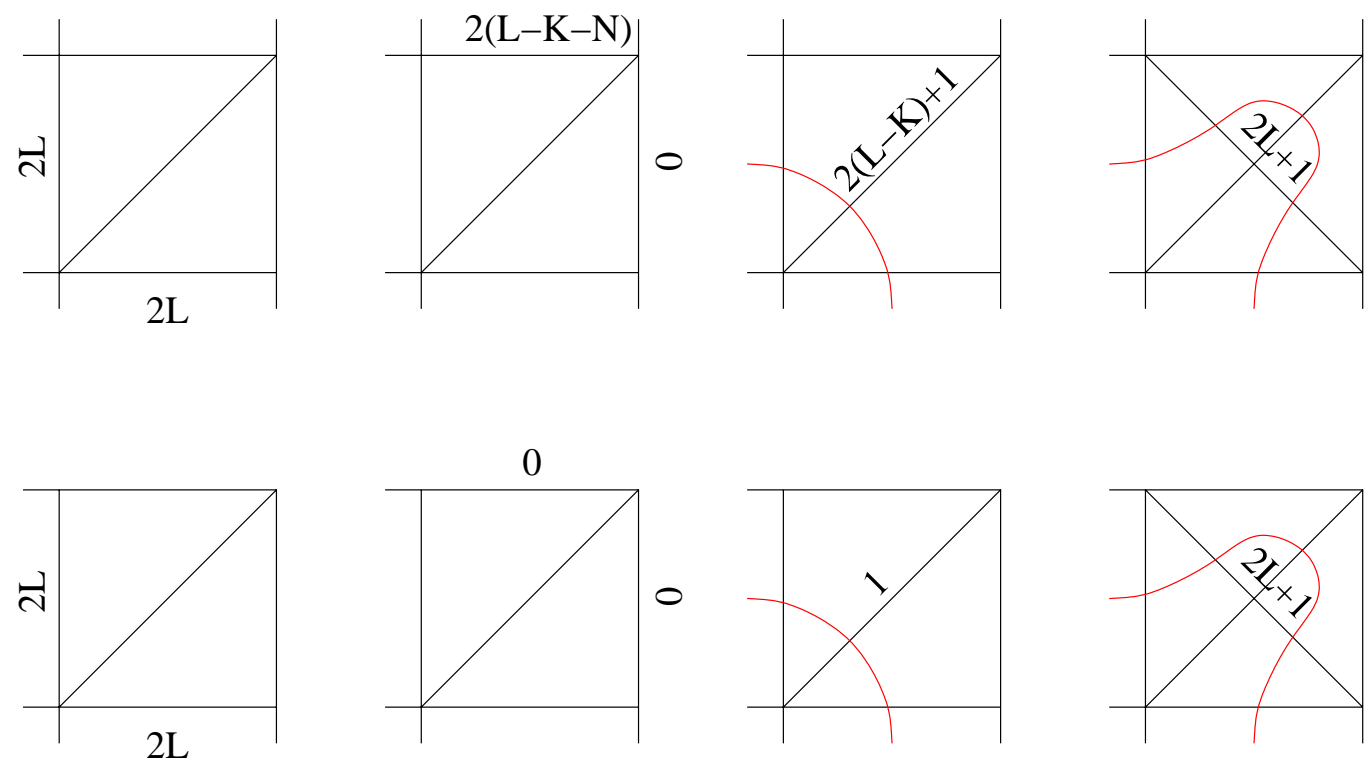

Figure 9: Orientations E: Local bisection keeps the curve connected.

The cases $\mathrm{E}$ can be treated as $\mathrm{A}-\mathrm{D}$ with the difference that boundary lines have level 0 and that we know that either $k$ or $l$ is of form $2^{L}$ or 0 which has the desired impact on the amount of factors of 2 . $\diamond$.

Remark: Note that (P1) mentions "subsequently traverses both children". This implies that the once a curve enters an element through its facet, it will not leave this element untill all its subsequently created descendents have been traversed, which is which makes the communication between processors "minimal" (see the figure to the right where the space-filling curve of elements is cut into four approximately equilength pieces).

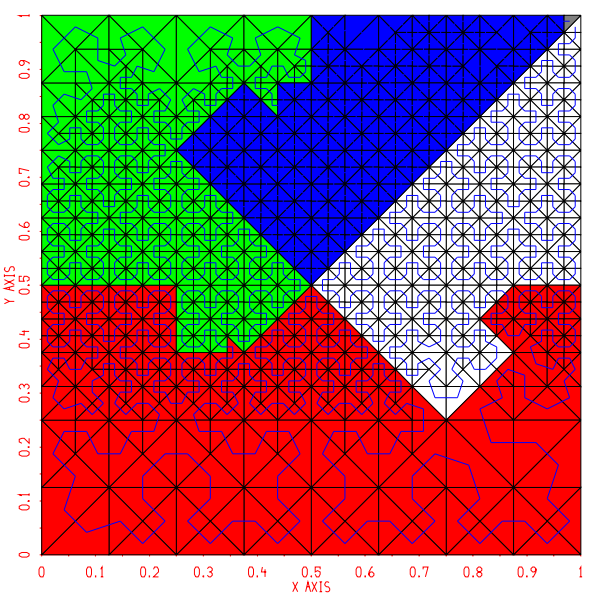

The obtained load-balance. 


\section{Extensions and 3-meshes}

The proof that the curve fulfills (P1) and (P2) remains valid if the coarse mesh is obtained under an invertable map $F$ from the standard coarse mesh in $[0,1]^{2}$. In this case one can extract the level information from the coordinates $F^{-1}(x)$ instead of from the coordinates $x$. For more information on possible extensions, see [6].

Also in the 3-dimensional case, compatible clusters are forecasted to be clusters in a uniform mesh (the uniform mesh where all elements have the same level as the elements in the cluster, see [6]). However, the determination of the amount of similar cluster orientations is not trivial. This, and whether our 2-factor-amount approach will still work for the extra symmetry lines, is currently under examination.

\section{Conclusions}

The local bisection refinement method from in [6] can be complemented with a local adaptation method which determines a curve for load balance purposes in the 2-d case. Both the local refinement and curve adaptation method are simple to formulate, simple to implement, and execute very efficiently. With the curve, the load-balancing is simple and communication is fast.

\section{References}

[1] E. Bänsch Local mesh refinement in 2 and 3 dimensions Impact of Computing in Science and Engineering, 3(1991), 181-191

[2] B. Joe and A. Liu On the shape of tetrahedra from bisection Math. Comp. 63(1944), 141-154

[3] I. Kossaczky A recursive approach to local mesh refinement in two and three dimensions Journal of Computational and Applied Mathematics, 55(1994), 275-288

[4] W. Layton, J.M. Maubach and P. Rabier Robustness of an elementwise parallel finite element method for convection diffusion problems SIAM Journal of Scientific Computing, 19(1998), 1870-1891 
[5] W. Layton, J.M. Maubach and P. Rabier Parallel algorithms for maximal monotone operators of local type Numerische Mathematik, 71(1995) $29-58$

[6] J. Maubach Local bisection refinement for n-simplicial grids generated by reflections SIAM Journal on Scientific Computing, 16(1995), 210-227

[7] J. Maubach The efficient location of simplicial neighbors for locally refined $n$-simplicial grids Proceedings of the 5-th International Meshing Roundtable 137-153, Pittsburgh, PA., USA, October 1996

[8] J. Maubach Iterative Methods for Non-Linear Partial Differential Equations ISBN 90-6196-421-0, C.W.I. Press, Amsterdam, The Netherlands, 1994

[9] J. Maubach Local bisection refinement and optimal order algebraic multilevel preconditioners, PRISM-97 conference Proceedings (eds. O. Axelsson et al), University of Nijmegen, the Netherlands, 1997, 121-136

[10] W.F. Mitchell Optimal multilevel iterative methods for adaptive grids SIAM Journal on Scientific and Statistical Computing, 13(1992), 146167

[11] A. Mukherjee An adaptive finite element code for elliptic boundary value problems in three dimensions with applications in numerical relativity Phd. Thesis, Penn State University, University Park, PA 16802, 1996

[12] Angel Plaza, José P. Suárez, Miguel A. Padrón, Sergio Falcón, Daniel Amieiro Mesh quality improvement and other properties in the fourtriangles longest-edge partition Comput. Aided Geom. Design 21(2004), 353-369

[13] M.C.Rivara and C. Levin A 3-d refinement algorithm suitable for adaptive and multi-grid techniques Journal of Computational and Applied Mathematics, 8(1992), 281-290 